\title{
Equatoiral winds on Saturn and the Stratospheric Oscillation
}

Liming $\mathrm{Li}^{1 *}$, Xun Jiang ${ }^{1}$, Andrew P. Ingersoll ${ }^{2}$, Anthony D. Del Genio ${ }^{3}$, Carolyn C. Porco ${ }^{4}$, Robert A. West ${ }^{5}$, Ashwin R. Vasavada ${ }^{5}$, Shawn P. Ewald ${ }^{2}$, Barney J. Conrath ${ }^{6}$, Peter J. Gierasch ${ }^{6}$, Amy A. Simon-Miller ${ }^{7}$, Conor A. Nixon ${ }^{8}$, Richard K. Achterberg ${ }^{8}$, Glenn S. Orton ${ }^{5}$ Leigh N. Fletcher ${ }^{9}$ Kevin H. Baines ${ }^{5}$

'Department of Earth and Atmospheric Sciences, University of Houston, Houston, TX, USA.

${ }^{2}$ Division of Geological and Planetary Sciences, Caltech, Pasadena, CA, USA.

${ }^{3}$ NASA Goddard Institute for Space Studies, New York, NY, USA.

${ }^{4}$ CICLOPS/Space Science Institute, Boulder, CO, USA.

${ }^{5}$ Jet Propulsion Laboratory, Caltech, Pasadena, CA, USA.

${ }^{6}$ Department of Astronomy, Cornell University, Ithaca, NY, USA.

${ }^{7}$ NASA Goddard Space Flight Center, Greenbelt, MD, USA.

${ }^{8}$ Department of Astronomy, University of Maryland, College Park, MD, USA.

${ }^{9}$ Atmospheric, Oceanic and Planetary Physics, University of Oxford, Oxford, UK

* To whom all correspondence should be addressed. E-mail: 1 li7@umail.uh.edu To be re-submitted to Nature-Geosciences, July 12, 2011 
The zonal jets on the giant planets are generally thought to be stable with time $\mathrm{e}^{1-3}$. Recently, there are still some debates about the general thought ${ }^{4-5}$. Here, we report a significant temporal variation of the equatorial jet at high-altitude on Saturn. Long-term (2004-2009) observations by Cassini reveal that wind speed at the 60 -mbar level increased from $270 \mathrm{~m} / \mathrm{s}$ in 2004 to $290 \mathrm{~m} / \mathrm{s}$ in 2008 , while the wind speed has been mostly constant over time at the 500-mbar level in the southern equatorial region. The Cassini observations further reveal that the equatorial jet intensified $\sim 60 \mathrm{~m} / \mathrm{s}$ in the stratosphere $(1-5 \mathrm{mbar})$ from 2005 to 2008. The fact that the wind acceleration is weaker at the $60-\mathrm{mbar}$ level $(\sim 20 \mathrm{~m} / \mathrm{s})$ than at the 1-mbar level $(\sim 60 \mathrm{~m} / \mathrm{s})$ demonstrates that the equatorial oscillation ${ }^{6-7}$ is damped when it propagates downwards to the tropopause around 60 mbar. The direct measurement of the varying equatorial jet around the tropopause also serves as a key boundary condition when deriving the thermal wind fields in the stratosphere.

Does the equatorial jet vary with time on the giant planets? A comparison ${ }^{4}$ of zonal winds between Voyager (1981-1982) and Hubble Space Telescope (1995-2002) observations suggested that velocity of Saturn's equatorial jet has decreased with time. Conversely, an analysis ${ }^{5}$ of observations by the Imaging Science Subsystem (ISS) on Cassini argued that the decrease of the equatorial jet discussed in the previous study ${ }^{3}$ could instead be explained by changes in altitude of the tracked clouds within a zonal wind structure with vertical shear. Studies ${ }^{8,9}$ based on data from the Composite Infrared Spectrometer (CIRS) on Cassini determine that vertical shear is present in the equatorial zonal winds. However, other studies ${ }^{10,11}$ suggest that the vertical shear is not strong enough to explain the wind decrease. The above debates reveal the need to further characterize the equatorial jet on Saturn with altitude and time. Such results also are critical for 
discriminating between different theories of large-scale circulation on the giant planets because they provide some important constraints on stability that must be fulfilled.

The equatorial thermal and dynamical structure of planetary atmospheres is also perturbed by quasi-periodic oscillations. A temperature oscillation with a period of 4-5 Earth years, known as the Quasi-Quadrennial Oscillation (QQO), was discovered in the equatorial stratosphere of Jupiter ${ }^{12}$. A recent study ${ }^{13}$ suggests that there may be a wind oscillation on Jupiter that is related to the temperature oscillation, similar to the Quasi-Biennial Oscillation (QBO) on Earth. Cassini observations ${ }^{4,5}$ recently showed a Semi-Annual Oscillation (SAO) of stratospheric temperature in the equatorial region of Satum. However, we have little information about the temporal variation of equatorial stratospheric winds. Such observations would not only enrich our knowledge of giant-planet dynamics but also help us explore the full phenomenology of Saturn's SAO.

Here we use long-term (2004-2009) observations from ISS and CIRS onboard Cassini to examine the temporal variations of the equatorial jet in both the troposphere and stratosphere of Saturn. In order to obtain accurate measurements of zonal winds in the equatorial region, we select only the ISS image pairs with relatively long time separations and high spatial resolutions to decrease the uncertainty of wind measurements. In addition, the selected ISS images contain long segments of the planetary limb so that they can be navigated well. The selected images on two dates (May 10, 2004 and December 30,2008) and the image processing steps are described in the Supplementary Information. Figure 1 shows examples of the processed maps at the strong methane filter (MT3) and the corresponding continuum filter (CB3). The MT3 observations are 
restricted to the hydrocarbon hazes or other photolysis products in the upper atmosphere ${ }^{11,14}$, which are $\sim 60$ mbar in the equatorial region of Saturn ${ }^{15}$. The CB3 observations are generally thought to represent ammonia clouds around 500 mbar. The map scale of Fig. 1 is $0.1 \%$ pixel in latitude and longitude, which corresponds to a spatial resolution of $\sim 105 \mathrm{~km} / \mathrm{pixel}$ at the equator. The region very close to the equator $\left(0-5^{\circ} \mathrm{S}\right)$ has low contrast due to the scattered sunlight from Saturn's rings. We identified few cloud features in this region, and exclude it from this study.

After selecting the ISS images, we searched for simultaneous thermal observations in the CIRS data. We found CIRS observations on December 29,2008 , nearly simultaneous to the ISS observations on December 30,2008 . Unfortunately, we did not find CIRS observations corresponding to the ISS observations on May 10, 2004. The closest CIRS observations of the equatorial region were taken on April 8,2005. Details of the selected CIRS observations and the procedure of temperature retrieval are also discussed in the Supplementary Information.

Based on the ISS and CIRS observations, we apply three methods to measure the zonal winds in the equatorial region of Saturn. The first method is a manual feature tracking. By tracking cloud features that do not substantially alter their shape over time, we can convert their movements into the zonal winds. The derived winds are averaged in $1^{\circ}$ latitude bins. The second method is similar to that of Limaye ${ }^{16}$, in which east-west strips of pixels are extracted from image pairs and shifted to find the maximal cross-correlation coefficient. The third method applies the thermal wind equation to the CIRS temperature maps, as described in our previous study9. We use winds measured from the ISS CB3 images to define the boundary level of the 
thermal wind integration, in order to maintain approximate consistence in time between the boundary condition and the CIRS temperature.

Wind measurements in Saturn's upper troposphere (i.e., $\sim 50-500$ mbar) are shown in Fig. 2. The uncertainty of wind measurements based on the ISS images comes from three sources: the standard deviation of multiple wind measurements, the optical characteristics of ISS cameras, and the accuracy of locating cloud/haze features in the ISS images. Uncertainty is discussed in the Supplementary Information. Panel A of Fig. 2 shows that the two image-based methods give a consistent result from the ISS CB3 images. The CB3 zonal wind near 500 mbar increased a few $\mathrm{m} / \mathrm{s}$ from 2004 to 2008, which is within the uncertainty of the measurement. Measurements on the ISS MT3 images (panel B), which probe the relatively high hazes around 60-mbar, reveal an increase of $\sim 20 \mathrm{~m} / \mathrm{s}$ from 2004 to 2008 around $12^{\circ} \mathrm{S}$, significantly larger than the uncertainty. We conclude that the wind speed at the level of the high-altitude hazes $(\sim 60$ mbar) increased, while the wind speed at the level of ammonia clouds ( 500 mbar) remained constant (within uncertainty) from 2004 to 2008.

To examine the temporal variation of zonal winds revealed by the ISS images, we further analyze the thermal winds in the equatorial region of Saturn. Panel B of Fig.2 shows the profile of thermally derived zonal winds at 60 mbar, which is based on Fig. S1 in the Supplementary Information. The 60 -mbar thermal winds also show an increase of $\sim 20 \mathrm{~m} / \mathrm{s}$ around $12^{\circ} \mathrm{S}$ between 2005 and 2008. The increase is mainly due to the warming of equatorial region and the corresponding changes of the temperature gradient in the troposphere of Saturn (Fig. S1). The uncertainty of thermal winds is closely related to the uncertainty of the retrieved temperature and 
the corresponding meridional gradient, which is difficult to estimate quantitatively. The uncertainty of the CIRS retrieved temperature is $\sim 1 \mathrm{~K}$ in the upper troposphere ${ }^{17}$, smaller than the magnitude of temperature variation of $\sim 2 \mathrm{~K}$ in the equatorial region shown in Fig. S1. The retrieval process leaves some uncertainty in the magnitude of warming around the equator, but the warming trend from 2005 to 2008 is robust ${ }^{17}$. The warming trend of atmospheric temperature intensifies the zonal winds via the thermal wind relationship, which explains the qualitative consistency of the high-altitude winds between the ISS measurements and the CIRS observations. On the other hand, the temperature of the relatively deep atmosphere around 500 mbar does not change significantly (Fig. S1), which is probably due to the long time scale of radiative adjustment of the deep atmosphere.

We also apply the thermal wind equation to the stratospheric temperature retrieved from the CIRS nadir observations (Fig. S2 in the Supplementary Information). Fig. S2 shows that the thermal winds in the stratosphere increased $\sim 60 \mathrm{~m} / \mathrm{s}$ from 2005 to 2008 , which confirms the previous results from the CIRS limb observations ${ }^{18}$. The temporal variation of the equatorial jet in the stratosphere is related to the varying temperature, which has been observed previously ${ }^{17-19}$. In these previous studies ${ }^{18,19}$, the varying temperature and the related thermal winds are further linked to the vertical propagation of Saturn's equatorial oscillation.

In this study, we report the temporal variation of equatorial jet speed at different altitudes in the troposphere and stratosphere of Saturn. We find that the wind speed of the equatorial jet around the tropopause $(\sim 60 \mathrm{mbar})$ increased $\sim 20 \mathrm{~m} / \mathrm{s}$ from 2004 to 2008 . This is the first change of the equatorial jet around the tropopause to be observed at the time scale of a few Earth years. 
At the time scale of one Saturn year ( $\sim 29.4$ Earth years), inter-annual variations of cloud morphology $\mathrm{y}^{20}$ and heat budget ${ }^{21}$ have been discovered on the giant planets. The season of Saturn will be the same in 2010-2011 as it was during the Voyager epoch (1980-1981). A detailed comparison of the wind field between the Cassini extended mission and the Voyager mission will shed light on the inter-annual variation of equatorial jet structure on Saturn.

As well as enriching our knowledge of the equatorial jet and oscillation, the temporal variation of zonal winds has important implications for atmospheric dynamics on Saturn. The high-altitude equatorial jet is at 60 -mbar, which is near the tropopause ${ }^{17}$. The direct measurement of zonal winds near the tropopause will benefit the exploration of thermal winds in stratosphere. Fouchet et al. ${ }^{4}$ and Guerlet et al. ${ }^{18}$ integrated the thermal wind equation in Saturn's low-latitude stratosphere by assuming a null wind as the boundary condition around 20 mbar. Obviously, measured winds near the tropopause will be a better boundary condition for deriving the thermal winds in the stratosphere, even though the different boundary conditions do not change the vertical structure of zonal winds discussed in the previous studies ${ }^{4,18}$. The weaker time variation of the equatorial jet at the $60-\mathrm{mbar}(\sim 20 \mathrm{~m} / \mathrm{s})$ than at the $1-\mathrm{mbar}(\sim 60 \mathrm{~m} / \mathrm{s})$ further implies a commonality in the vertical propagation of the equatorial oscillation between Saturn and Earth ${ }^{22}$. Finally, the difference in behavior in the time variation of zonal winds between the troposphere (e.g., 500-mbar) and the stratosphere (e.g., I-mbar) suggests that the driving mechanisms of zonal winds are probably different between the stratosphere, where the radiative effects are important and the troposphere where the convection and turbulence are important. 


\section{References}

1. Irwin, P. G. J. in Giant planets of our solar system, 141-142 (Praxis Publishing, 2003).

2. Cho, J. Y., Polvani, L. M., The morphogenesis of bands and zonal winds in the atmospheres on the giant outer planets. Science 19, 335-337 (1996).

3. Galperin, B., Sukoriansky, S., Huang, H. Universal $n^{-5}$ spectrum of zonal flows on giant planets. Physics of Fluids 13, 1545-1549 (2001).

4. Sanchez-Lavega, A., Perez-Hoyos, S., Rojas, J. F. \& French, R. G. A strong decrease in Saturn's equatorial jet at cloud level. Nature 423, 623-625 (2003).

5. Porco, C. C. et al. Cassini imaging science: Initial results on Saturn's atmosphere. Science $307,1243-1247(2005)$.

6. Fouchet, T., Guerlet, S., Strobel, D. F., Simon-Miller, A. A., Bezard, B. \& Flasar, F. M. An equatorial oscillation in Saturn's middle atmosphere. Nature 453, 200-202 (2008).

7. Orton, G. S. et al. Semi-annual oscillations in Saturn's low-latitude stratospheric temperatures. Nature 453, 196-199 (2008).

8. Flasar, F. M. et al. Temperatures, winds, and composition in the Saturnian system. Science 307, 1247-1251 (2005).

9. Li, L., Gierasch, P. J., Achterberg, R. K., Conrath, B. J., Flasar, F. M., Vasavada, A. R., Ingersoll, A. P., Banfield, D., Simon-Miller, A. A. \& Fletcher, L. N. Strong jet and a new thermal wave in Saturn's equatorial stratosphere. Geophys. Res. Lett. 35, L23208 (2008).

10. Perez-Hoyos, S. \& Sanchez-Lavega, A. On the vertical wind shear of Saturn's equatorial jet at cloud level. Icarus 180, 161-175 (2006). 
11. Sanchez-Lavega, A., Hueso, R. \& Perez-Hoyos, S. The three-dimensional structure of Saturn's equatorial jet at cloud level. Icarus 187, 510-519(2007).

12. Leovy, C. B., Friedson, A.J. \& Orton, G. S. The quasi-quadrennial oscillation of Jupiter's equatorial atmosphere. Nature 354, 380-382 (1991).

13. Simon-Miller, A. A. \& Gierasch, P. J. On the Long-Term Variability of Jupiter's Winds and Brightness as Observed from Hubble. Icarus 210, 258-269 (2010).

14. García Melendo, E., Sánchez..Lavega, A., Rojas, J. F., PérezhHoyos, S. \& Hueso, R. Vertical shears in Saturn's eastward jets at cloud level. Icarus 201, 818-820 (2009).

15. Garcia-Melendo, E., Sanchez-Lavega, A., Legarreta, J., Perez-Hoyos, S. \& Hueso, R. A strong high altiude narrow jet detected at Saturn's equator, Geophys. Res. Lett. 37, L22204 $(2010)$

16. Limaye, S. S. Jupiter: new estimates of the mean zonal flow at the cloud level. Icarus $\mathbf{6 5}$, 335-352(1986).

17. Fletcher, L. N., Achterberg, R. K., Greathouse, T. K., Orton, G. S., Conrath, B. J., SimonMiller, A. A., Guerlet, S., Irwin, P. G. J. \& Flasar, F. M. Seasonal Change on Saturn from Cassini/CIRS Observations, 2004-2009. Icarus 208, 337-352 (2010).

18 Guerlet, S., Fouchet, T., Bezard, B., Flasar, F. M., Simon-Miller, A. A. Evolution of the equatorial oscillation in Saturn's atmosphere between 2005 and 2010 from Cassini/CIRS limb data analysis. Geophys. Res. Lett. 38, L09201 (2011).

19 Schinder, P. J., Flasar, F. M., Marouf, E. A., French, R. G., McGhee, C. A., Kliore, A. J., Rappaport, N. J., Barbinis, E., Fleischman, D., Anabtawi, A. Saturn's equatorial oscillation: evidence of descending thermal structure from Cassini radio occultations. Geophys. Res. Lett. 38, L08205 (2011). 
20. Baines, K. H. et al. Polar lightning and decadall..sscale cloud variability on Jupiter. Science 318, 226-229 (2007).

21. Li, L., Conrath, B., Gierasch, P., Achterberg, R., Nixon, C., Simon-Miller, A. A., Flasar, F. M., Banfield, D., Baines, K., West, R., Vasavada, A., Mamoutkine, A., Segura, M., Bjoraker, G., Orton, G., Fletcher, L., Irwin, P. \& Read, P. Emitted power of Saturn. J. Geophys. Res. 115, E11002 (2010).

22. Baldwin, M. P., et al. The Quasi-biennial oscillation. Reviews of Geophysics 39, 179-229 (2001). 


\section{Correspondence}

To whom all correspondence and requests for materials should be addressed to Dr. Li with Email:1li7@mail.uh.edu

\section{Acknowledgements}

NASA Cassini Data Analysis Program (CDAP) funded this work. We acknowledge Enrique Garcia-Melendo, Ricardo Hueso, Agustin Sanchez-Lavega, and Santigo Perez-Hoyos for providing valuable comments and constructive discussions. We are also grateful for valuable comments and suggestions on this work from two anonymous reviewers.

\section{Author contributions}

L. L. measured the ISS winds (correlated), computed the thermal winds, and conceived the overall research. X. J. carried the ISS wind measurements (cloud-tracking). X. J., A. P. I., A. D. D. G., C. C. P., R. A. W., A. R. V., S. P. E., B. J. C., P. J. G., A. A. S-M, C. A. N., R. K. A., G. S. O., L. N. F., and K. H. B. provides assistance in interpreting the observational results. 


\section{Figure Captions}

Figure 1. Southern equatorial maps based on ISS multi-filter images taken in May 10, 2004. The spatial resolution of the cylindrical maps is $\sim 105 \mathrm{~km} / \mathrm{pixel}$ (please refer to Table $\mathrm{Sl}$ in the Supplementary Information). (A) MT3 map at time 1 (t1). (B) MT3 map at time 2 (t2), which is taken by ISS in 9.5 hours later than t1. (C) and (D) are the same as (A) and (B) except for the ISS images taken at filter CB3.

Figure 2. Saturn wind measurements on two different dates. (A) Low-altitude winds from the ISS/CB3 images. The CB3 winds are located at or deeper than the 500-mbar pressure level, and used as the boundary condition of thermal winds. (B) High-altitude winds from the ISS/MT3 images and the CIRS thermal observations. The CIRS thermal winds at 60 mbar are taken from the tropospheric thermal winds in the Supplementary Information. The horizontal solid lines and dashed lines are the uncertainties for the cloud-tracking winds and the correlation winds, respectively. 
Figure 1

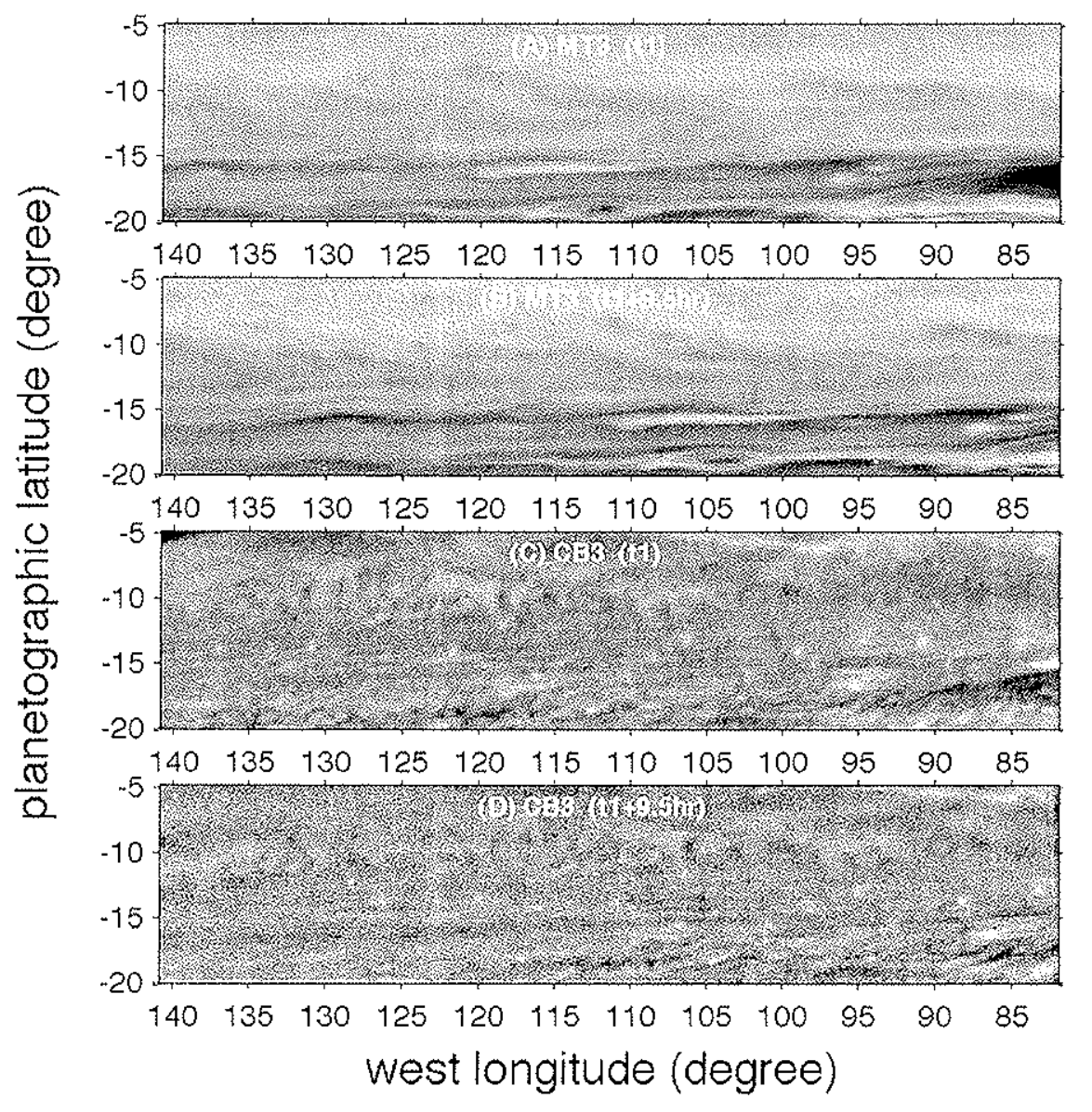


Figure 2

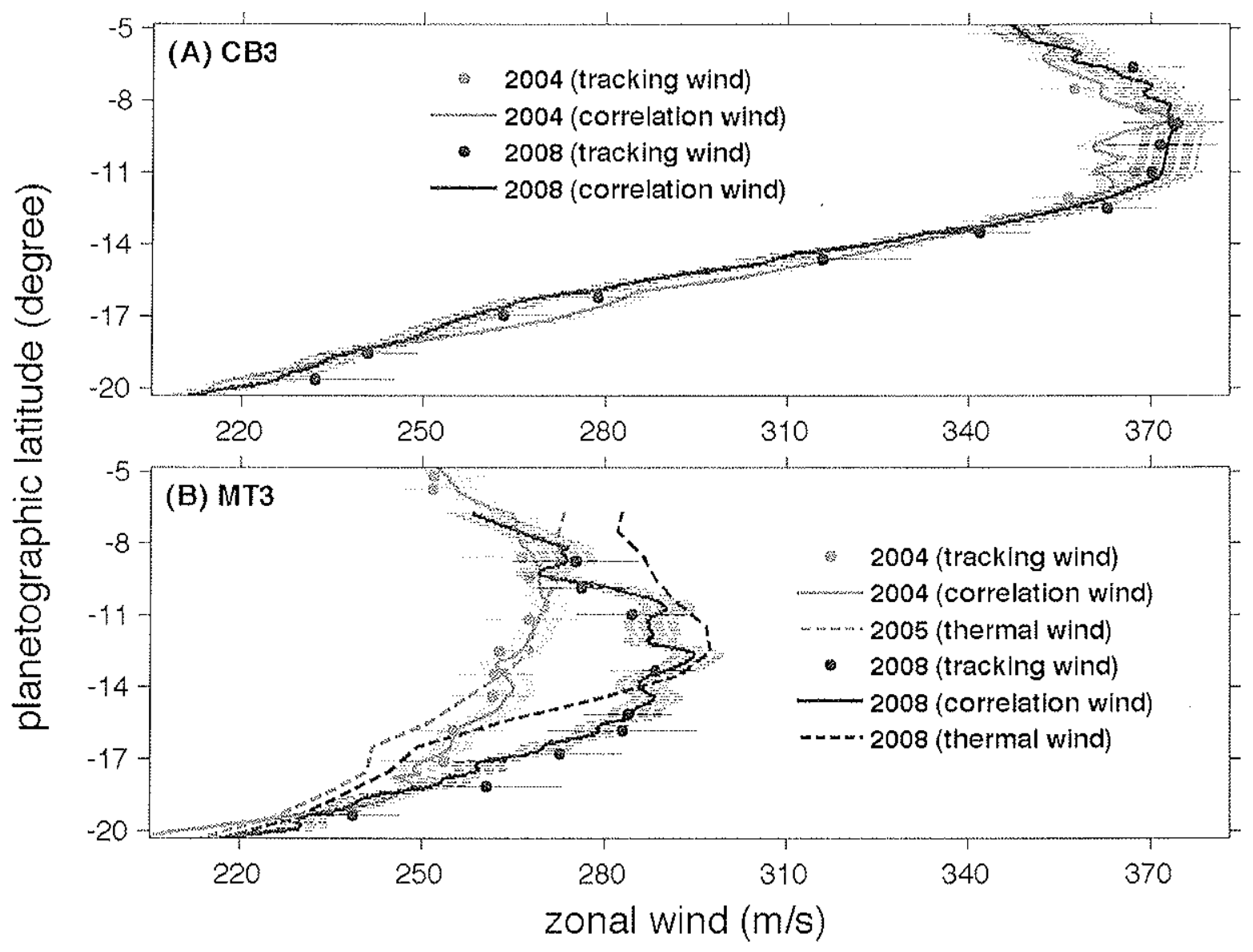

